\title{
Réaction du greffon contre l'hôte et antigènes d'histocompatibilité mineurs
}

La greffe de moelle osseuse est un traitement de plus en plus fréquemment proposé aux malades souffrant de leucémies ou d'aplasies médullaires. La réaction aiguë du greffon contre l'hôte (graft versus host, GVH) est secondaire à l'attaque des cellules du receveur présentant des antigènes mineurs d'histocompatibilité par les lymphocytes cytotoxiques du donneur. La déplétion du greffon en cellules $T$ diminue l'incidence de la GVH mais aussi l'effet antileucémique de la greffe. Les progrès futurs consisteront en des essais de dissociation entre l'effet antileucémique et la GVH. A la GVH aiguë succède souvent une GVH chronique qui semble liée à l'activation de clones $\mathrm{CD}^{+}$autoréactifs stimulés, sous l'action de cytokines, par l'hyperexpression de molécules de classe II du CMH à la surface des cellules autologues. La tolérance immunologique qui succède enfin à ces phases de GVH implique des mécanismes qui sont encore mal compris d'« anergie clonale », c'est-à-dire d'inactivation des cellules cytotoxiques auto- ou alloréactives.

\section{Claude Perreault Robert Bélanger Martin Gyger Sylvie Brochu}

\section{RÉFÉRENCES}

1. Bortin MM, Rimm AA. Increasing utilization of bone marrow transplantation. Transplantation 1986 ; 42 : 229-34.

2. Gale RP, Reisner Y. Graft rejection and graft-versus-host disease: mirror images. Lancet 1986; 1 : 1468-70.

\section{ADRESSE}

C. Perreault : docteur en médecine, chercheur boursier-clinicien du FRSQ. R. Bélanger : docteur en médecine, chercheur boursier du FRSQ. M. Gyger : docteur en médecine, professeur titulaire. Université de Montréal. S. Brochu: licenciée ès sciences, boursière du Conseil de la recherche médicale du Canada. Hôpital Maisonneuve-Rosemont, département d'hématologie, 5415, boulevard de l'Assomption, Montréal, Québec, HIT 2M4 a greffe de moelle osseuse (GMO) allogénique chez l'homme suscite un intérêt considérable. Le nombre de transplantations effectuées chaque année augmente de façon exponentielle et la création de l'International Bone Marrow Transplant Registry témoigne des remarquables efforts de collaboration consentis par la communauté scientifique à l'échelle mondiale [1]. Deux éléments expliquent cet intérêt. Pour l'hématologiste, la GMO représente le traitement de choix, et parfois la seule intervention thérapeutique possible, pour des maladies autrement mortelles comme l'aplasie médullaire sévère, les leucémies aiguës, la leucémie myéloöde chronique et diverses maladies congénitales touchant les cellules lymphoïdes et/ou myéloïdes. Pour l'immunologiste, la GMO représente le seul type de transplantation où nous obtenons régulièrement un état de tolérance spécifique. Six mois après la greffe, la majorité des patients ne prend plus d'immunosuppresseurs et le greffon tolère le receveur.

Malgré les progrès réalisés durant les dernières années, la GMO demeure cependant une approche thérapeutique complexe réservée à un nombre restreint d'individus. Règle générale, elle n'est proposée qu'aux candidats jeunes (moins de 40 à 50 ans) possédant un donneur HLA-identique (ce que l'on ne rencontrera que dans $25 \%$ de la population). Même à l'intérieur de ce groupe restreint, la GMO comporte des risques appréciables puisque environ $25 \%$ des receveurs décèderont à la suite de complications précoces ou tardives reliées à la transplantation. Ces limites et échecs sont avant tout imputables au conflit immunologique bidirectionnel provoqué lors de la transplantation [2]. D'une part, les cellules lymphoïdes résiduelles du receveur peuvent rejeter le greffon médullaire et, d'autre part, les lymphocytes du greffon peuvent attaquer le receveur, provoquant ainsi une réaction du greffon contre l'hôte 
(graft versus host, GVH). Après une GMO conventionnelle (c'est-à-dire lorsqu'on n'élimine aucune cellule du greffon), le rejet est un événement rare qui survient précocement et se manifeste par une aplasie médullaire irréversible. En revanche, la GVH est un phénomène fréquent qui peut survenir trois semaines à deux ans après la transplantation et dont lés manifestations cliniques sont polymorphes. Dans le texte qui suit, nous tenterons de présenter une mise au point des connaissances sur la GVH en insistant sur les acquisitions récentes concernant sa physiopathologie, tout en limitant notre discussion à la situation généralement rencontrée en clinique humaine, c'est-àdire la GMO allogénique entre individús possédant les mêmes antigènes majeurs d'histocompatibilité (HLA chez l'homme) mais dont les antigènes mineurs sont différents.

\section{Les antigènes mineurs d'histocompatibilité}

Les antigènes d'histocompatibilité sont des antigènes allotypiques membranaires capable de provoquer un rejet ou une GVH. Par exclusion, tous ceux qui ne font pas partie du complexe majeur d'histocompatibilité $(\mathrm{CMH})$ sont dits « mineurs » [3]. Cette dénomination est discutable puisque l'immunogénicité de l'ensemble des antigènes mineurs est équivalente à celle du CMH [4]. Comme personne n'a réussi à produire d'anticorps contre les antigènes mineurs, nous ne savons pratiquement rien sur leur structure biochimique et leur distribution. Ils sont nombreux, plus de 40 loci chez la souris [3], leur polymorphisme allélique est restreint [5] et certains sont peut-être produits par des rétrovirus endogènes [6]. On croit que ce sont de petits peptides ( 9 à 25 acides aminés) qui, après leur synthèse dans le cytoplasme, sont liés de façon non covalente aux antigènes $\mathrm{CMH}$ de classe I et ainsi présentés à la surface de la cellule[7]. Les antigènes mineurs ne sont reconnus que par les lymphocytes $T$ et non les lymphocytes B [8]. Leur étude se heurte à deux difficultés supplémentaires. Le même antigène peut être codé par des loci différents d'une lignée de souris à l'autre [9] . In vivo et in vitro, la $\mathrm{m} / \mathrm{s} n^{\circ} 1$ vol. 6, janvier 90 réponse aux antigènes mineurs est fonction non seulement du génotype $\mathrm{CMH}$ et extra-CMH des cellules répondeuses, mais aussi d'interactions positives et négatives complexes difficilement prévisibles [10]. Ainsi la présence d'un antigène mineur A sur une cellule cible peut augmenter ou diminuer la réponse à un antigène $B$ présent sur la même cellule et codé par un locus indépendant.

\section{Manifestations cliniques de la GVH}

La GVH peut prendre deux formes, aiguë ou chronique. La GVH aiguë survient durant les 100 premiers jours qui suivent la greffe. Les principaux organes cibles sont la peau et les muqueuses (érythème, épidermolyse), le tube digestif (stomatite, diarrhées) et le foie (choléstase et cytolyse)[11]. La GVH chronique apparaît 100 à 700 jours après la greffe. Elle peut toucher pratiquement tous les tissus et organes à l'exception du myocarde et des reins, et ses manifestations sont très polymorphes, prenant l'apparence de diverses maladies auto-immunes comme la sclérodermie, le lupus érythémateux disséminé, le syndrome de Sjögren, la myasthénie grave et le purpura thrombopénique autoimmun [12]. La GVH est fréquemment associée à une pancytopénie qui peut être centrale (stroma médullaire et/ou cellules souches hémopoïétiques) ou périphérique (cellules sanguines matures). Dans la forme chronique, on retrouve fréquemment des auto-anticorps comme dans les maladies auto-immunes «spontanées ». GVH aiguë et chronique sont fréquemment associées. La plupart des patients qui présentent une GVH chronique ont présenté une GVH aiguë et vice versa. Si la GVH est une complication sérieuse, parfois mortelle, ce n'est pas surtout à cause des lésions directement infligées aux différents organes cibles, mais à cause du déficit immunitaire qui lui est associé. La GVH et son traitement freinent le développement du système immunitaire transplanté et rendent ainsi le patient très sensible aux infections [13]. Certains paramètres cliniques sont associés à une incidence accrue de GVH : receveur (et donneur ?) plus âgé, combinaison receveur de sexe masculin et donneuse non nullipare, infection bactérienne ou virale latente ou manifeste. Deux approches sont utilisées pour prévenir la GVH. La plus répandue consiste à administrer au receveur des immunosuppresseurs comme le méthotrexate et la ciclosporine durant six mois [14]. Elle n'est que partiellement efficace puisque environ $50 \%$ des patients présenteront une GVH aiguë et/ou chronique. De plus, les immunosuppresseurs utilisés sont hépatotoxiques et néphrotoxiques. L'autre approche consiste à éliminer les lymphocytes $\mathrm{T}$ du greffon, étant donné que ces cellules sont à l'origine de la GVH (voir ci-après). Il est possible d'éliminer $99 \%$ des cellules $\mathrm{T}$ en utilisant des lectines ou des anticorps monoclonaux et ainsi de diminuer considérablement le risque de GVH. Malheureusement, l'élimination des lymphocytes $\mathrm{T} d u$ greffon n'améliore pas la survie des patients car elle est associée à deux complications majeures, une augmentation du taux de rejet et une perte de l'effet antileucémique du greffon (greffon versus leucémie; GVL) [15]. Si la GMO est si efficace pour réduire le risque de récidive néoplasique, c'est en grande partie grâce à l'effet GVL dont la perte peut facilement faire doubler le taux de rechute. Il nous est actuellement impossible de prévenir la GVH sans perdre l'effet GVL ni augmenter le risque de rejet [16].

\section{La GVH aiguë, une agression allo-immune}

De nombreuses études ont démontré que les lymphocytes $T$ matures contenus dans le greffon sont à la fois nécessaires et suffisants pour induire une GVH [17]. Les cellules T qui sont produites par les cellules souches du greffon n'ont pas ce potentiel. Chez la souris, le rôle des sous-populations $T$ varie en fonction des lignées étudiées [18, 19]. Dans la majorité des cas, les cellules $\mathrm{CD}^{+}$ jouent un rôle prédominant ou exclusif. Dans un premier temps, elles reconnaissent comme étrangers les antigènes mineurs présents à la surface des macrophages et cellules dendritiques du receveur [20]. Une fois sensibilisées, les cellules $\mathrm{CD}^{+}$ 


\section{RÉFÉRENCES}

3. Loveland B, Simpson E. The non-MHC transplantation antigens : neither weak nor minor. Immunol Today 1986; 7 : 223-9. 4. Graff RJ. Minor histocompatibility genes and their antigens. In : Morse HC, ed. Origins of Inbred Mice. New York: Academic Press, $1978: 371-89$.

5. Rammensee HG, Klein J. Polymorphism of minor histocompatibility genes in wild mice. Immunogenetics $1983 ; 17: 637-47$.

6. Colombo M, Jaenisch $\mathrm{R}$, Wettstein PJ. Endogenous retroviruses lead to the expression of a histocompatibility antigen detectable by skin graft rejection. Proc Natl Acad Sci USA 1987 ; 84 : 189-93.

7. Towsend A, Bodmer $\mathrm{H}$. Antigen recognition by class I-restricted T lymphocytes. Ann Rev Immunol 1989; 7 : 601-24.

8. Simpson E. T and B lymphocytes: two repertoires or one? Immunol Letters 1986 ; 12 : 185-93.

9. Wettstein PJ. Immunodominance in the T cell response to multiple non- $\mathrm{H}-2$ histocompatibility antigens. IV. Partial tissue distribution and mapping of immunodominant antigens. J Immunol 1987 ; 139 : 2166-71.

10. Halle-Pannenko O, Mary JY, Motta R. Sex-dependent effects of non-H-2 loci on lethal GVH reaction induced across an $\mathrm{H}-2$ barrier. Immunogenetics 1986; 217 : 217-24.

11. Perreault C, Gyger M, Boileau J, et al. Acute graft-versus-host disease after allogeneic bone marrow transplantation. Can Med Assoc J 1983 ; 129 : 969-74.

12. Sullivan KM, Shulman HM, Storb R, et al. Chronic graft-versus-host disease in 52 patients : adverse natural course and successful treatment with combination immunosuppression. Blood $1981 ; 57$ : 267-76.

13. Lum LG. The kinetics of immune reconstitution after human marrow transplantation. Blood 1987 ; 69 : 369-80.

14. Neudorf S, Filipovich A, Ramsay N, Kersey J. Prevention and treatment of acute graftversus-host disease. Semin Hematol 1984; 21 : 91-100.

15. Gale RP. T cells, bone marrow transplantation and immunotherapy : use of monoclonal antibodies, immune interventions in disease. Ann Intern Med 1987 ; 106 : 257-74.

16. Butturini A, Gale RP. T cell depletion in bone marrow transplantation for leukemia: current results and future directions. Bone Marrow Transplant 1988; 3 : 185-92.

17. Korngold R, Sprent J. Lethal GVHD across minor histocompatibility barriers: nature of the effector cells and role of the $\mathrm{H}$ 2 complex. Immunol Rev 1983 ; 71 : 5-29. 18. Korngold R, Sprent J. Variable capacity of L3T $4^{+} \mathrm{T}$ cells to cause lethal graft-versushost disease across minor histocompatibility barriers in mice. J Exp Med 1987 ; 165 : 155264.

19. Hamilton BL. L3T4-positive T cells participate in the induction of graft-vs-host disease in response to minbr histocompatibility antigens. J Immunol 1987; 139 : 2511-5. 20. Perreault $C$, Pelletier $M$, Bélanger $R$ Gyger M, David M, Bonny Y, d'Angelo G. The role of host bone marrow-derived cells in graft-versus-host disease. Int J Cell Cloning 1986 ; 4 (suppl. 1) : 189-90.

21. Cleveland MG, Annable CR, Klimpel

GR. In vivo and in vitro production of IFN $\beta$ and IFN- $\alpha$ during graft-vs-host disease. attaquent les différentes cellules de l'hôte porteuses de ces antigènes mineurs comme les kératinocytes, entérocytes et hépatocytes. La présentation des antigènes mineurs et leur reconnaissance par les cellules $\mathrm{CD}^{+}$ suivent le principe de restriction par le $\mathrm{CMH}$. Ainsi Korngold a démontré que les cellules $\mathrm{CD}^{+}$reconnaissent uniquement les antigènes mineurs associés aux antigènes $\mathrm{CMH}$ de classe I. Alors que dans certaines lignées de souris les cellules $\mathrm{CD} 4^{+}$ne sont pas impliquées dans l'induction de la GVH, dans plusieurs lignées elles peuvent potentialiser l'agression par les cellules $\mathrm{CD}^{+}$et, dans de rares cas, provoquer une GVH aiguë sans la collaboration des lymphocytes CD8 $8^{+}$. Nous ne savons pas si ces cellules $\mathrm{CD} 4^{+}$reconnaissent les antigènes mineurs en association avec les antigènes $\mathrm{CMH}$ de classe II.

Les lymphocytes $\mathrm{CD} 8^{+}$et $\mathrm{CD} 4^{+}$peuvent provoquer des lésions tissulaires par cytolyse directe ou en sécrétant trois variétés de lymphokines, l'interféron $\beta$ [21], le tumor necrosis factor (TNF) [22] et l'interleukine-2 [23]. La sécrétion de ces lymphokines a de nombreux effets biologiques dont le principal semble être l'activation de cellules tueuses naturelles dont l'activité cytotoxique n'est pas restreinte par le CMH [24] (figure 1).

\section{La GVH chronique, une maladie auto-immune}

Les travaux de Parkman chez la souris C57BL/6 recevant un greffon médullaire de souris LP suggèrent que la GVH chronique est une maladie auto-immune dépendante des clones de lymphocytes $\mathrm{CD}^{+}$autoréactifs qui sont stimulés par les antigènes $\mathrm{CMH}$ de classe II communs au couple donneur-receveur [25]. Nous possédons très peu de renseignements sur l'émergence et le devenir de ces cellules autoréactives. De Clerck a démontré qu'elles produisent des lymphokines capables de stimuler la production de collagène par les fibroblastes, entraînant ainsi une fibrose qui est caractéristique des lésions de GVH chronique [26]. Pourquoi la GVH chronique, une maladie auto-immune, survient-elle habituellement chez les receveurs qui ont présenté une GVH aiguë, qui est une maladie allo-immune? Nous avons vu que lors d'une GVH aiguë, les cellules effectrices produisent des lymphokines. Deux d'entre elles, l'interféron et le TNF, augmentent l'expression des antigènes $\mathrm{CMH}$ de classe II sur les cellules qui en possèdent déjà et entraînent l'apparition « inappropriée » de ces antigènes sur des cellules qui, normalement, ne les expriment pas (par exemple, kératinocytes et entérocytes). Cette expression inappropriée des antigènes CMH de classe II stimulerait la prolifération des cellules $\mathrm{CD}^{+}$autoréactives.

\section{Mécanismes de la tolérance}

A la suite d'une GMO, environ la moitié des receveurs ne développent pas de GVH et, chez la plupart de ceux qui sont atteints, la GVH disparaît après administration d'immunosuppresseurs sur une longue période. Nous réussissons donc à obtenir, dans la majorité des cas, un état de tolérance spécifique sans qu'il soit nécessaire de poursuivre les immunosuppresseurs. Cet objectif n'est pratiquement jamais atteint en transplantation rénale, cardiaque ou hépatique.

Les mécanismes responsables du maintien de l'état de tolérance varient en fonction du modèle étudié, un des paramètres cruciaux étant le degré de maturité des cellules $T$ impliquées. On doit donc distinguer la tolérance au soi, qui se développe normalement chez le fotus, et la tolérance post-transplantation qui, elle, est induite chez un individu immunologiquement mature. La tolérance au soi repose sur des mécanismes centraux (thymiques) et périphériques (extrathymiques), alors que seuls ces derniers sont impliqués dans la tolérance post-transplantation.

Plusieurs peptides du soi sont présents dans le thymus. La tolérance à ces antigènes est attribuable à une délétion clonale, les cellules autoréactives étant éliminées dans le thymus au stade $\mathrm{CD}^{+} \mathrm{CD}^{+}$(voir Lexique Immunologie, suppl. au $n^{\circ} 1$, vol. 5, p. 25). Ainsi la reconnaissance d'un complexe antigène mineurantigène $\mathrm{CMH}$ du soi par le récepteur d'un thymocyte entraîne l'élimination de ce dernier par apop- 
tose* [27]. Le phénomène de la délétion clonale n'implique que les peptides du soi, d'origine endogène ou exogène, présents à la surface des cellules dendritiques de la médulla thymique.

La tolérance aux antigènes du soi qui ne sont pas présents dans le thymus et la tolérance à l'ensemble des antigènes rencontrés en transplantation dépendent uniquement de mécanismes périphériques. Cette tolérance d'origine extra-thymique est due non pas à une élimination, mais à une inactivation des lymphocytes $T$ matures impliqués. Deux mécanismes ont été proposés, l'anergie clonale et la suppression. Dans le premier cas, les lymphocytes $\mathrm{T}$ sont comme paralysés sans que l'on sache exactement de quelle façon [28, 29]. La présence constante du tolérogène semble essentielle au maintien de cette anergie clonale, qui peut être supprimée par injection d'interleukine-2. Dans d'autres modèles, l'inactivation semble dépendre de cellules suppressives dont on distingue deux variétés principales, les cellules de type veto et les suppresseurs antiidiotypiques. Soit «A » un lymphocyte $T$ reconnaissant un antigène sur une cellule «B». Dans le modèle anti-idiotypique, le suppresseur «C» est un lymphocyte $T$ qui inhibe la réaction $\mathrm{A}$ anti- $\mathrm{B}$ après avoir reconnu le récepteur $\mathrm{A}$ anti-B à la surface des cellules A [30]. Dans le modèle veto, la cellule B elle-même inactivera les cellules A qui reconnaissent les antigènes présents à sa surface [31]. Il n'est pas encore possible d'évaluer l'importance relative des différents mécanismes périphériques dans le maintien de la tolérance aux antigènes mineurs suite à une GMO allogénique [32].

\section{L'effet GVL}

La plupart des candidats à une GMO souffrent de leucémie aiguë ou chronique. La GMO allogénique constitue l'approche la plus efficace dont nous disposions pour éliminer un

\footnotetext{
* Apoptose : voir l'article de Pierre Golstein (m/s no 8, vol. 5, p. 546); il s'agit d'une mort cellulaire induite par un signal extracellulaire et comportant une fragmentation nucléaire, une dégradation de l'ADN, etc.

$\mathrm{m} / \mathrm{s} n^{\circ} 1$ vol. 6, janvier 90
}

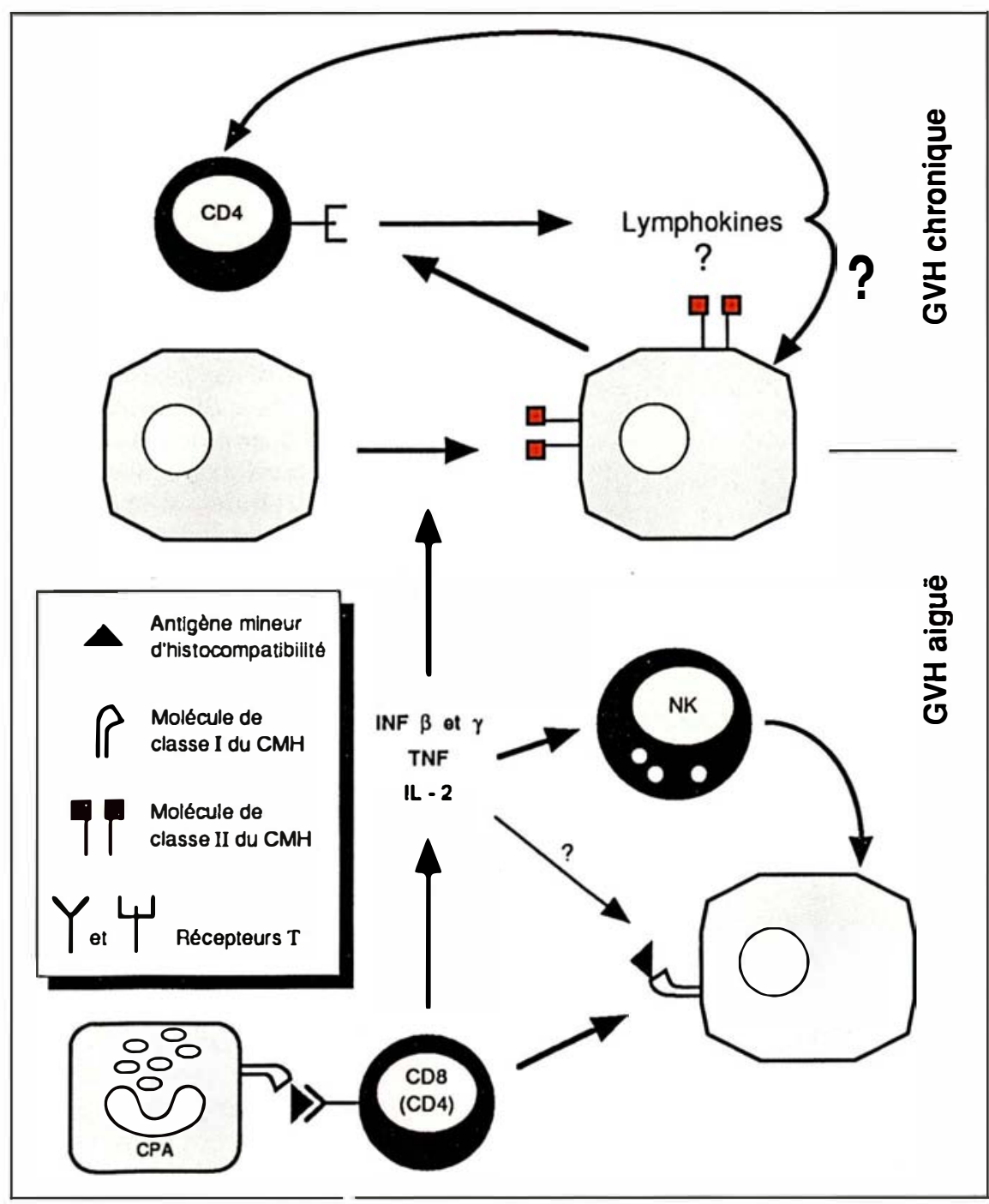

Figure 1. Les cellules impliquées dans la GVH. Les cellules au cytoplasme pâle appartiennent au receveur et celles dont le cytoplasme est foncé au donneur. Les grandes cellules polygonales sont les cellules cibles de la GVH (kératinocytes, hépatocytes, entérocytes). CPA = cellule présentatrice d'antigènes.

clone leucémique. Si dans ce contexte le taux de récidive néoplasique est faible, c'est non seulement parce que nous administrons des doses élevées de chimiothérapie et/ou radiothérapie au receveur, mais aussi parce que l'injection de lymphocytes allogéniques est associée à un effet antileucémique aussi important que mal compris. La reconnaissance d'un effet GVL repose sur trois observations [33]. Le taux de récidive leucémique est plus faible après une GMO allogénique que syngénique. Chez les individus qui reçoivent un greffon allogéni- que, le taux de rechute leucémique est moins élevé chez ceux qui présentent une GVH. L'élimination des lymphocytes $\mathrm{T}$ d'un greffon allogénique augmente la probabilité de récidive

Certains auteurs ont supposé que l'effet GVL était relié à un effet antinéoplasique de certaines lymphokines (interféron, TNF) produites au cours d'une GVH et/ou à l'activation des cellules tueuses naturelles qui s'ensuit. Les travaux de Tutschka chez le rat vont à l'encontre de cette hypothèse [34] . Si on effectue une GMO chez des rats de lignée $A B$ 


\section{RÉFÉRENCES}

22. Piguet PF, Grau GE, Allet B, Vassalli P. Tumor necrosis factor/cachectin is an effector of skin and gut lesions of the acute phase of graft-vs-host disease. J Exp Med 1987; 166 : 1280-9.

23. Ferrara JLM, Marion A, McIntyre JF, Murphy GF, Burakoff SJ. Amelioration of acute graft-vs-host disease due to minor histocompatibility antigens by in vivo adminis tration of anti-interleukin 2 receptor antibody. J Immunol 1986; 137 : 1874-7.

24. Ferrara JLM, Guillen FJ, Van Dijken PJ, Marion A, Murphy GF, Burakoff SJ. Evidence that large granular lymphocytes of donor origin mediate acute graft-versus-host disease. Transplantation $1989 ; 47: 50-4$

25. Parkman R. Clonal analysis of murine graft-vs-host disease. I. Phenotypic and functional analysis of $\mathrm{T}$ lymphocyte clones. $J$ Immunol 1986 ; 136 : 3543-8.

26. De Clerck Y, Draper V, Parkman R. Clonal analysis of murine graft-vs-host disease. II. Leukokines that stimulate fibroblast proliferation and collagen synthesis in graft-vshost disease. I Immunol 1986 ; 136 : 3549-52. 27. Von Boehmer H, Teh HS, Kisielow P. The thymus selects the useful, neglects the useless and destroys the harmful. Immuno Today $1989 ; 10$ : 57-61.

28. Morahan G, Allison J, Miller JFAP. Tolerance of class I histocompatibility antigens expressed extrathymically. Nature $1989 ; 339$ : $622-4$.

29. Rammensee HG, Kroschewski R, Frangoulis $\mathrm{B}$. Clonal anergy induced in mature $\mathrm{V} \beta 6+\mathrm{T}$ lymphocytes on immunizing Mls$1^{\mathrm{b}}$ mice with Mls-1 ${ }^{\mathrm{a}}$ expressing cells. Nature $1989 ; 339: 541-4$

30. Batchelor JR, Lombardi G, Lechler RI Speculations on the specificity of suppression. Immunol Today 1989; 10 : 37-40.

31. Ishikawa $H$, Hino $T$, Kato $H$, Suzuki $H$, Saito K. Cytotoxic T lymphocyte response to minor $\mathrm{H}-42^{\mathrm{a}}$ alloantigen in $\mathrm{H}-42^{\mathrm{b}}$ mice : clonal inactivation of the precursor cytotoxic $\mathrm{T}$ lymphocytes by veto-like spleen cells that express the $\mathrm{H}-42^{\mathrm{a}}$ antigen. J Immunol 1986 $137: 2080-8$

32. Perreault C, Bélanger R, Gyger M, Allard A, Brochu S. The mechanism of graft-hosttolerance in murine radiation chimeras transplanted across minor histocompatibility barriers. Bone Marrow Transplant 1989; 4 : 837.

33. Butturini A, Bortin MM, Gale RP. Graftversus-leukemia following bone marrow transplantation. Bone Marrow Transplant $1987 ; 2: 233-42$

34. Tutschka PJ, Berkowitz SD, Tuttle S Klein J. Graft-versus-leukemia in the rat. The antileukemic efficacy of syngeneic and allogeneic graft-versus-host disease. Transplant Proc 1987 ; 19 : 2668-73.

35. Truitt RL, Ash RC. Manipulation of Tcell content in transplanted human bone marrow : effect on GVH and GVL reactions. In: Truitt RL, Gale RP, Bortin MM, eds. Cellular Immunotherapy of Cancer. New York : Alan R Liss Inc., 1987 : 409-21. en leur injectant un greffon A ou B et que deux semaines plus tard on injecte au receveur des cellules leucémiques de type $B$, tous les receveurs présenteront une GVH, mais on détectera un effet GVL uniquement chez ceux dont le greffon médullaire provenait de donneurs A. Autrement dit, l'effet GVL ne se manifeste que si les cellules leucémiques sont allogéniques par rapport au greffon. Les travaux de Truitt chez la souris suggèrent que l'effet GVL dépend de deux types de lymphocytes $T$ cytotoxiques. Les premiers peuvent tuer à la fois des cellules allogéniques normales et des cellules leucémiques alors que les autres ne tuent que les cellules leucémiques [35]. L'existence de lymphocytes $\mathrm{T}$ cytotoxiques reconnaissant exclusivement les cellules leucémiques laisse entrevoir la possibilité théorique de dissocier la GVH et l'effet GVL. A ce stade, le problème fondamental est d'identifier de façon précise les antigènes responsables de la spécificité antileucémique de ces İymphocytes cytotoxiques. Bien qu'a priori cette observation semble plaider en faveur de l'existence d'antigènes spécifiques à la surface des cellules néoplasiques, la différence cruciale entre le phénotype des cellules leucémiques et celui des cellules normales est peut-être quantitative plutôt que qualitative. En effet, on sait qu'in vitro les cellules néoplasiques sont plus sensibles que des cellules normales à l'activité des lymphocytes cytotoxiques dirigés contre des antigènes d'histocompatibilité majeurs ou mineurs (c'est-à-dire non néoplasiques). Il est logique de supposer que cette sensibilité particulière est due à l'expression d'une plus grande quantité d'antigènes d'histocompatibilité à la surface des cellules néoplasiques. $\mathrm{Ce}$ phénomène pourrait expliquer l'apparente spécificité antileucémique d'une partie des cellules cytotoxiques responsables de l'effet GVL.

\section{Conclusion}

L'objectif à atteindre en transplantation médullaire est clair, prévenir la GVH sans perdre l'effet GVL ni augmenter le taux de rejet. Dans cette optique, les questions suivantes nous semblent mériter une attention particulière. Quelle est la structure bio- chimique des antigènes mineurs d'histocompatibilité, par quels gènes sont-ils codés et quels mécanismes en contrôlent l'expression ? Quels sont les antigènes cibles de l'effet GVL? Peut-on favoriser l'expansion des clones de lymphocytes $\mathrm{T}$ cytotoxiques qui reconnaissent exclusivement les cellules néoplasiques? Quelle est l'importance relative des différents mécanismes périphériques dans la tolérance qui fait suite à une GMO allogénique?

\section{Summary}

Graft versus host disease and minor histocompatibility antigens

When bone marrow transplantation is performed across minor histocompatibility barriers, graftversus-host disease (GVH) remains the major problem. The acute phase of GVH clearly represents an allogeneic attack mediated by donor - derived $\mathrm{T}$ cells that recognize host minor antigens. These minor antigens are small peptides bound to class I major histocompatibility antigens. Chronic GVH appears to be an autoimmune disease mediated by $\mathrm{CD}^{+} \mathrm{T}$ cells that proliferate when stimulated by autologous class II major histocompatibility antigens. Tolerance to host antigen is mediated by peripheral (extra-thymic) mechanisms involving clonal anergy or suppression of alloreactive cells. While GVH per se has a negative impact on recipient survival, the associated graft-versus-leukemia effect greatly decreases the leukemic relapse rate. New strategies should be developped to prevent GVH without jeopardizing the graft-versus-leukemia effect nor increasing the rejection rate.

\section{TIRÉS A PART}

C. Perreault. 